\title{
Predicting survival after targeted therapy for mRCC
}

Despite the widespread use of molecular targeted agents (MTAs) such as antiangiogenics (for example, sorafenib and sunitinib) and mammalian target of rapamycin inhibitors (for example, everolimus and temsirolimus) for patients with metastatic renal cell carcinoma (mRCC), few studies have specifically assessed criteria for monitoring response and outcome predictors for these therapies.

\section{4 ...TGR can be implemented very easily as it relies on standard CT scans... 77}

Two studies published recently in European Urology have shed new light on this issue. In the first study, Ferte et al. describe the inadequacies of the Response Evaluation Criteria in Solid Tumors (RECIST) criteria in the mRCC setting and demonstrate the benefits of using tumour growth rate (TGR) to predict outcomes, by evaluating medical records at Gustave Roussy clinic, France.

According to these authors, MTAs often stabilize disease, rather than shrink tumours, meaning that RECIST sums (the longest diameters of the targeted lesions) can provide an inaccurate representation of disease burden. By comparison, TGR estimates changes in tumour volume over time, generating a quantitative and dynamic evaluation of response.

Ferte et al. calculated TGRs for clinically relevant periods (before MTA introduction, first cycle, at progression, and after discontinuation) for all patients in the Treatment Approaches in Renal Cancer Global Evaluation Trial (TARGET; $n=903$ ) and found that, not only did TGR permit an earlier and more precise evaluation of drug response than RECIST, it was also strongly associated with progression-free and overall survival.

"A few alternatives to RECIST have been proposed, but all are lacking supporting evidence or necessitate additional exams; TGR can be implemented very easily as it relies on standard CT scans," says Charles Ferte. "Combining TGR and MSKCC score could be of major help at the bedside."

The investigators also found that TGR revealed drug-specific profiles at disease progression. For patients treated with sorafenib, but not everolimus, TGR at progression was

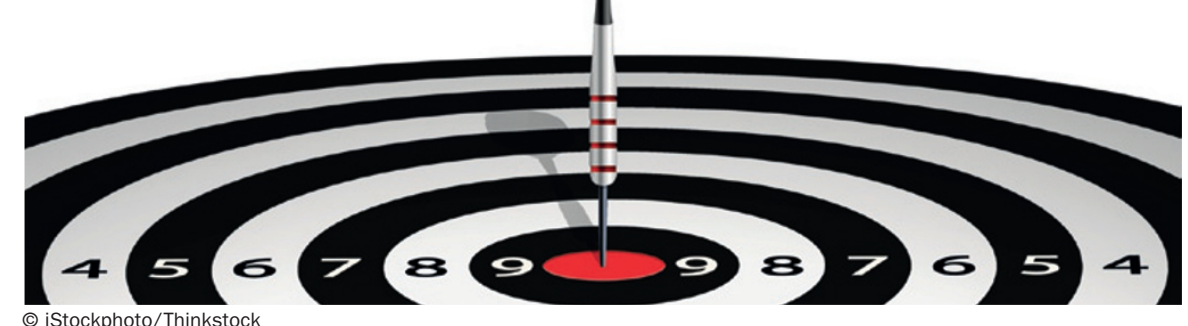

significantly lower than before treatment. Plus, an apparent 'flare-up' after sorafenib discontinuation suggested that some cells were still sensitive to sorafenib at the time of progression. "Alternative discontinuation schemes (perhaps similar to those used for $\beta$-blockers or corticosteroids) should perhaps be investigated," suggests Ferte.

For the second study, McKay et al. investigated the effect of bone and liver metastases on overall survival and timeto-treatment failure for 2,027 patients with mRCC treated with MTAs, using data from the International Metastatic Renal Cell Carcinoma Database Consortium (IMDC).

When compared with other metastatic sites and adjusted for IMDC risk factors, hazard ratios were 1.4 for bone metastases, 1.42 for liver metastases, and 1.82 for both. "Not only is metastatic site prognostic, in that patients with bone or liver metastases have worse survival, it could also be predictive, as some agents target the bone, which is a difficult site to monitor and treat," says Rana McKay.

Melanie Clyne 